\title{
Antifungal Streptomyces spp., Plausible Partners for Brood-Caring of the Dung Beetle Copris tripartitus
}

\author{
Sung Hun Kim, Goeun Park, Jin-Soo Park * (D) and Hak Cheol Kwon *(D) \\ Natural Product Informatics Research Center, Korea Institute of Science and Technology (KIST), \\ Gangneung 25451, Gangwon-do, Korea; shkim12@wooree.co.kr (S.H.K.); 092297@kist.re.kr (G.P.) \\ * Correspondence: jinsoopark@kist.re.kr (J.-S.P.); hkwon@kist.re.kr (H.C.K.)
}

\begin{abstract}
The dung beetle Copris tripartitus Waterhouse (Coleoptera: Scarabaeidae) is a coprophagous insect that lives in and feeds primarily on the feces of mammalian herbivores and is known to protect their offspring from the pathogen-rich environment by performing parental care for brood balls. Brood balls under continuous management by dung beetle are rarely contaminated by entomopathogenic fungi compared to abandoned brood balls. On the supposition that dung beetles may benefit from mutualistic bacteria that protect their offspring against fungal pathogens, we evaluated the antifungal activities of bacteria isolated from the dung beetle and brood ball. As a result, bacterial isolates, mainly streptomycetes, manifested potent and broad-spectrum antifungal activity against various fungi, including entomopathogens. Of the isolates, Streptomyces sp. AT67 exhibited pronounced antifungal activities. Culture-dependent and independent approaches show that this strain has occurred continuously in dung beetles that were collected over three years. Moreover, metabolic profiling and chemical investigation demonstrated that the strain produced an antifungal polyene macrocyclic lactam, sceliphrolactam, as a major product. Our findings imply that specific symbiotic bacteria of $C$. tripartitus are likely to contribute brood ball hygiene by inhibiting fungal parasites in the environment.
\end{abstract}

Citation: Kim, S.H.; Park, G.; Park, J.-S.; Kwon, H.C. Antifungal Streptomyces spp., Plausible Partners for Brood-Caring of the Dung Beetle Copris tripartitus. Microorganisms 2021, 9, 1980. https://doi.org/10.3390/ microorganisms 9091980

Academic Editor: Patricia Luis

Received: 17 June 2021

Accepted: 14 September 2021

Published: 17 September 2021

Publisher's Note: MDPI stays neutral with regard to jurisdictional claims in published maps and institutional affiliations.

Keywords: dung beetle; Copris tripartitus; DGGE; insect-symbionts; antifungal agents

\section{Introduction}

Dung beetles are environmentally important for the recycling of organic matter in ecosystems by the decomposition of feces, but they are threatened by the widespread use of agrochemicals and habitat destruction due to changes in agriculture and urbanization. The dung beetle Copris tripartitus Waterhouse (Coleoptera: Scarabaeidae) was registered in the list of endangered insects in Korea and is only found in certain areas, such as Jeju island, where pastures are abundant for stock-farming [1,2]. The species is paracoprid and builds a nest in the soil underneath the dung pat and brings dung for feeding and breeding into the nest [2,3]. The adult dung beetle puts considerable effort into constructing brood balls for oviposition from the collected dung pieces because the environment surrounding them is unfavorable for survival and maintenance of broods [4]. In addition, the adult females stay with the brood balls until the successful emergence of the offspring [5]. On the other hand, orphaned broods who do not receive continuous care are vulnerable to parasite invasions, which eventually lead to the destruction of the egg or larva inside the brood ball [6,7]. Many studies have attempted to understand a way for the dung beetle to protect offspring via brood care, but an obvious host defense mechanism remains to be elucidated $[8,9]$.

Insects have protected themselves and their offspring against pathogens and parasites by adopting various strategies because antagonists could significantly affect their survival and development [10]. Among the strategies, host-bacterial mutualism is effective and feasible, and is frequently found in various insects, such as ants, wasps, bees, and beetles [11-14]. The symbiotic bacteria, mainly actinomycetes, engage in the protection of their 
host or their food resources by means of antimicrobial substances [13,15-17]. As symbionts, they are often associated with hygiene improvement by inhibiting competing microorganisms. In this regard, we hypothesized that the symbiotic bacteria of $C$. tripartitus may play a role as a defensive agent for the survival of offspring in brood balls against parasites and pathogens. Of the many diverse symbionts, microbiome members in the digestive system were chosen because preparation of brood balls by dung beetles is accompanied by physiological processes, including chewing, regurgitation, and defecation, indicating that the bacterial flora of the brood ball could be closely associated with that of the beetle's digestive system. There are already some clues supporting our hypothesis. First, the dung beetle Onthophagus taurus (Coleoptera: Scarabaeidae) can vertically transmit their specific endosymbiotic microbiome to their offspring through the brood ball, and the unidentified matrix materials and microbes were only found in the brood ball prepared by the female in sterile rearing conditions [18]. Second, several actinomycetes have been often isolated from the dung beetle $C$. tripartitus, and they produced secondary metabolites with various chemical structures and biological activities [19-23].

To specify the symbiotic bacteria beneficial to the brood care of the dung beetles $C$. tripartitus, we used a dual-culture assay, screening bacterial isolates with antifungal activity from $C$. tripartitus. In addition, we examined whether the antifungal bacteria are observed continually in dung beetles through the culture-independent approach over two years. Furthermore, we identified an antifungal metabolite of the specified endosymbiont by combined secondary metabolite profiling and bioactivity-guided.

\section{Materials and Methods}

\subsection{Sample Collection}

Dung beetles were collected from pastures in Jeju Island, Korea $\left(33^{\circ} 38^{\prime} \mathrm{N}, 126^{\circ} 73^{\prime} \mathrm{E}\right)$. The beetles were sampled every August from 2012 to 2014. They were caught alive using dung-baited pitfall traps without preservative [24]. Soil and cattle dung samples were also obtained from the collection site. Brood balls, each containing a larva (approximately 3rd larval instar) of dung beetles reared under laboratory conditions were provided by the National Institute of Agricultural Sciences, Republic of Korea [25].

\subsection{Microbial Isolation and Identification}

Female beetles $(n=3)$ collected in 2012 were surface sterilized with 70\% ethanol for $5 \mathrm{~min}$, followed by a rinse with sterile distilled water. Each was dissected to obtain the digestive tract from the whole body. The outer layer of the brood balls $(n=2)$ was carefully scraped off to obtain a portion between the outer surface and the egg chamber. The prepared samples were then ground and suspended in saline solution. The sample suspensions were heat-shock treated at $55^{\circ} \mathrm{C}$ for $10 \mathrm{~min}$ to stimulate germination of the actinomycete spores. Samples were then inoculated by serial dilution plating on four selective agar plates containing nalidixic acid $(20 \mu \mathrm{g} / \mathrm{mL})$, A1 agar (A1A, $10 \mathrm{~g}$ soluble starch, $2 \mathrm{~g}$ yeast extract, $4 \mathrm{~g}$ peptone, $18 \mathrm{~g}$ agar in $1 \mathrm{~L}$ distilled water), Actinomycete isolation agar (AIA), Czapek-Dox agar (CDA), and Potato-Dextrose agar (PDA). During incubation at $25^{\circ} \mathrm{C}$ for three weeks, bacterial and fungal strains were isolated in pure culture and maintained on YME agar (YMEA, $4 \mathrm{~g}$ yeast extract, $10 \mathrm{~g}$ malt extract, $4 \mathrm{~g}$ dextrose, $18 \mathrm{~g}$ agar in $1 \mathrm{~L}$ distilled water) and PDA until further processing. The isolated strains were identified based on their morphological characteristics and molecular data. Genomic DNA (gDNA) of the bacterial strains was extracted using a QIAamp DNA Mini Kit (Qiagen, Hilden, Germany) and then used as a DNA template for PCR with primers 27F and 1492R to amplify the partial 16S rRNA gene [26]. The isolation of the DNA from fungal mycelia was performed using a DNeasy Plant Mini Kit (Qiagen). The extracted DNA was used as a template for amplification of the fungal ITS region using primers ITS1 and ITS4 [27]. The resultant PCR products were purified using a QIAquick PCR purification kit (Qiagen). The amplified DNA were ligated with pGEM-T easy vector (Promega) and transformed into Escherichia coli DH5 $\alpha$. The purified plasmids were sequenced on an ABI 
3730XL DNA Sequencer (Applied Biosystems, Foster City, CA). Nucleotide sequences were edited and assembled using the BioEdit program [28]. The sequences were compared using the BLASTN (https: / / blast.ncbi.nlm.nih.gov/Blast.cgi, accessed on 10 May 2021.) for performing similarity searches from rRNA/ITS databases consisting of 16S ribosomal RNA of bacterial and archaea type strains [29]. For phylogenetic analysis, each sequence was aligned using the ClustalW multiple sequence alignment program [30]. The phylogenetic tree was constructed using the Maximum Likelihood (ML) method applied to pairwise sequence distances calculated by the general time reversible (GTR) model with the MEGA program [31,32]. In addition, partial rpoB gene of antifungal isolates was obtained by PCR amplification using a primer set for rpoB-P2 [33] and purification, and sequenced. Additional phylogenetic tree of the concatenated sequences of $16 \mathrm{~S}$ rRNA and rpoB genes was constructed using the ML method and GTR model.

\subsection{Antifungal Screening by the Dual Culture Assay}

The bacterial strains isolated from dung beetles and brood balls were primarily screened for antifungal activity against ubiquitous soil fungi (AF1: Aspergillus fumigatus ATCC 26,430 and AF2: Aspergillus flavus ATCC 16883) and entomopathogenic fungi (AF3: Beauveria bassiana ATCC 7159 and AF4: Metarhizium anisopliae ATCC 20500) by the dual culture assay. The assay was conducted as follows. Each bacterial strain was cultivated in $10 \mathrm{~mL}$ of YME/TSB with shaking at $250 \mathrm{rpm}$ for three days at $28{ }^{\circ} \mathrm{C}$ and then harvested by centrifugation. The obtained cell paste was washed twice with distilled water and re-suspended in $100 \mu \mathrm{L}$ of fresh medium [34]. Of the concentrated cell suspension, $20 \mu \mathrm{L}$ was inoculated onto the center of YME agar plates and incubated for three weeks at $25^{\circ} \mathrm{C}$. After incubation, two agar plugs $(6 \mathrm{~mm}$ diameter) with growing mycelium of the test-fungi grown on PDA plates were placed on both sides $15 \mathrm{~mm}$ away from the resident colony (Figure 1a). After seven days, the antifungal activity was determined by measuring the distance between the edges of the fungal mycelium and the antagonistic bacterium. Subsequently, the isolates with antagonistic activity against at least one of the four tested fungi were also tested against the seven fungi isolated from the brood balls in the same manner as above. The experiment was repeated three times to confirm reproducibility.

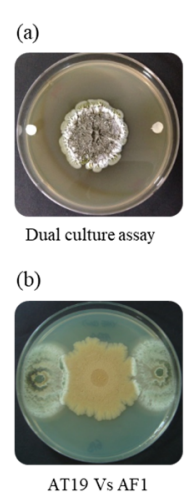

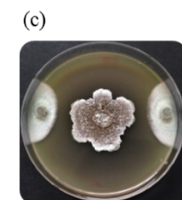
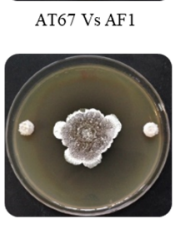

AT67 Vs AF5

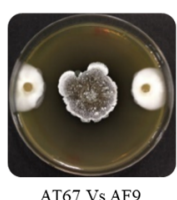

AT67 Vs AF9

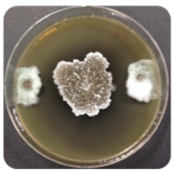

AT67 Vs AF2

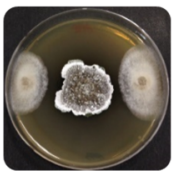

AT67 Vs AF6

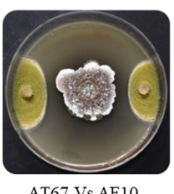

AT67 Vs AF 10

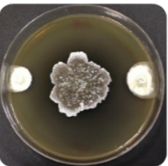

AT67 Vs AF3

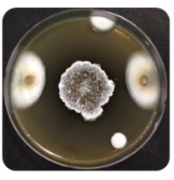

AT67 Vs AF7

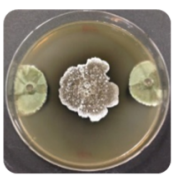

AT67 Vs AF11

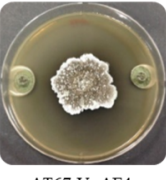

AT67 Vs AF4

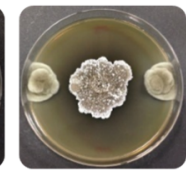

AT67 Vs AF8

Figure 1. Dual culture antifungal assays with Actinobacteria isolated from dung beetles (Copris tripartitus) or their dung balls. (a) The experimental figure of the dual culture assay, (b) Representative result of bacterial isolate with no antifungal activity (e.g., AT19 vs. AF1), (c) Antifungal activity of strain AT67 against 11 fungi (AF1-AF11).

\subsection{Extraction of Nucleic Acids from Samples}

The dung beetles (12 females and 12 males) collected in 2013 and 2014 were surface sterilized and dissected to obtain their digestive tract. Each tissue sample was homogenized 
in lysis buffer (20 mM Tris-Cl, $2 \mathrm{mM}$ sodium EDTA, 1.2\% Triton X-100 and $20 \mathrm{mg} / \mathrm{mL}$ lysozyme at a $\mathrm{pH}$ of 8.0 ) with a $5 \mathrm{~mm}$ stainless steel bead using a TissueLyser (Qiagen). The DNA from the homogenates was isolated using the QIAamp DNA Mini Kit (Qiagen) according to the manufacturer's instructions. The prepared brood ball $(n=8)$ and cattle dung $(n=3)$ samples were homogenized with glass beads by vortexing and then centrifuged at 14,000 rpm for $1 \mathrm{~min}$ to pellet stool particles. The supernatants were used for DNA extraction with the use of the QIAamp DNA Stool Mini Kit (Qiagen). DNA extraction from the habitat soil samples $(n=2)$ was performed using the PowerMax ${ }^{\circledR}$ Soil DNA Isolation Kit (MO BIO, Carlsbad, CA, USA) following the manufacturer's protocol. The extracted DNA samples were purified using PowerClean DNA Clean-up kit (MO BIO) to eliminate PCR inhibitors. The final DNA extracts were checked for purity and concentration using a NanoDrop spectrometer ND-1000 (NanoDrop Technologies, Wilmington, DE, USA) and stored at $-20{ }^{\circ} \mathrm{C}$ until further processing.

\subsection{Nested PCR Amplification}

Nested polymerase chain reaction (nested PCR) was applied for specific and sensitive amplification of the target genes in the community. The amplification of the V6/V7 region of the 16S rRNA genes from the extracted DNA samples was performed using specific primer sets for the genus Streptomyces, including the isolates with antifungal activity against tested fungi. The Actinomycetes-specific primers 243F and 1378R (Table S1) were used as described by Heuer et al. [35] in the first round PCR. The second PCR was performed with internal primers 984F/GC (containing a GC clamp [36]) and DBstR (Table S1) as follows: $95{ }^{\circ} \mathrm{C}$ for $3 \mathrm{~min}$, followed by 25 cycles of $30 \mathrm{~s}$ at $95^{\circ} \mathrm{C}, 20 \mathrm{~s}$ at $54{ }^{\circ} \mathrm{C}$, and $30 \mathrm{~s}$ at $72{ }^{\circ} \mathrm{C}$, with a final extension for $7 \mathrm{~min}$ at $72{ }^{\circ} \mathrm{C}$. The PCR amplification yielded fragments of approximately $227 \mathrm{bp}$ in size. The primer DBstR was constructed based on the alignment of the 16S rRNA gene sequences of 15 Streptomyces isolates and their closest phylogenetic relatives in this study. The PCR products were diluted to the same concentration (500 ng DNA $/ \mu \mathrm{L}$ ) using a NanoDrop spectrometer ND-1000 (NanoDrop Technologies) and used for DGGE analysis.

\subsection{DGGE Analysis}

The existence of the antifungal isolates in the samples derived from dung beetles was studied by denaturing gradient gel electrophoresis (DGGE) analysis with the DCode ${ }^{\mathrm{TM}}$ Universal Mutation Detection System (Bio-Rad Laboratories, Hercules, CA, USA). For rapid identification, the DGGE marker was constructed by mixing the $16 \mathrm{~S}$ rRNA gene fragments of the five strains (strains AT20, AT67, AT92, AT95, and AT99) amplified with primer sets 1 and 2 (Figure S1 and Table S1) and were co-electrophoresed with the samples. In the DGGE analysis, the PCR products were loaded onto $10 \%(w / v)$ polyacrylamide gels $(40 \%$ acrylamide/bis solution, 37.5:1, Bio-Rad) with a linear gradient of 40-65\% denaturant (100\% denaturant consisted of $7 \mathrm{M}$ urea and $40 \%(v / v)$ formamide). The gels were run at $60 \mathrm{~V}$ for $18 \mathrm{~h}$ in $1 \times$ TAE buffer ( $40 \mathrm{mM}$ Tris-acetate, $1 \mathrm{mM}$ EDTA, pH 8.3) kept at a constant temperature of $60^{\circ} \mathrm{C}$. After electrophoresis, the gels were stained with ethidium bromide $(\mathrm{EtBr})$ for $30 \mathrm{~min}$ and then photographed under a UV transilluminator.

\subsection{Identification of DGGE Bands}

The DGGE bands that migrated at the same positions with respect to the DGGE marker were identified by directly sequencing the re-amplified DNA from the bands. The bands stained with SYBR Green I were excised using a sterile surgical blade on UltraBright LED Transilluminator (Maestrogen, Las Vegas, NV, USA). The excised bands were homogenized using a disposable homogenizer and then purified using the QIAquick gel extraction kit (Qiagen). The purified DNA samples were re-amplified by PCR with the primers 984F and DBstR (Table S1) and were sequenced. 


\subsection{HPLC analysis of Culture Extracts}

The 16 antifungal strains were subjected to chemical analysis to profile their secondary metabolites. Each strain was cultured in YME liquid medium with constant shaking at $28^{\circ} \mathrm{C}$ for seven days. The culture broths were extracted with ethyl acetate and analyzed using an Agilent 1200 series LC/MS system (Agilent, Wilmington, USA) equipped with a Phenomenex Luna C18(2) column $(150 \times 4.6 \mathrm{~mm}, 5 \mu \mathrm{m})$. The mobile phase was eluted with a linear aqueous acetonitrile gradient from 10 to $100 \%$ in $0.05 \%$ formic acid at a flow rate of $0.7 \mathrm{~mL} / \mathrm{min}$. All samples were injected at the same concentration $(1 \mathrm{mg} / \mathrm{mL})$ in the $\mathrm{LC} / \mathrm{MS}$ system. The obtained HPLC chromatograms were compared with BioNumerics software (Version 7.5; Applied-Maths, Sint-Martens-Latem, Belgium). After 16 chromatograms were processed (baseline subtraction and smoothing), they were analyzed using the Dice similarity coefficient based on the presence/absence of the peaks and clustered by UPGMA (unweighted pair-group method averages) cluster analysis [37].

\subsection{Culture and Extraction of Strain AT67}

Strain AT67 was cultured aerobically in YME liquid medium $(25 \mathrm{~mL})$ with shaking at $200 \mathrm{rpm}$ for three days at $28^{\circ} \mathrm{C}$. The seed culture was transferred into $1 \mathrm{~L}$ Erlenmeyer flasks containing $500 \mathrm{~mL}$ of YME liquid medium with 1 percent $(v / v)$ inoculums. The culture flasks $(16 \mathrm{~L})$ were incubated at $28^{\circ} \mathrm{C}$ on an orbital shaker at $200 \mathrm{rpm}$ for 14 days. The non-ionic macroreticular resin, Amberlite XAD-7 (20 g/L, Sigma), was employed in the extraction of organic material from the culture broth of strain AT67. The adsorbed resin was washed with deionized water and then extracted in acetone. The acetone extract was concentrated on a rotary evaporator under reduced pressure.

\subsection{Separation and Bioassay of Bioactive Fraction}

For identification of antifungal metabolites produced by strain AT67, the culture extract was subjected to $\mathrm{SiO}_{2}$-column chromatography (Kieselgel 60, 0.063-0.200 mm, Merck) with hexane/ethyl acetate and ethyl acetate/methanol gradients as eluents to yield five fractions, F1-F5. The antifungal activity of each fraction was determined by their minimal inhibitory concentration (MIC) values against fungal strains AF10 and AF11 closely related to $A$. flavus and $M$. anisopliae, respectively. The crude extract and fractions were diluted by serial dilutions with DMSO, ranging from 0.002 to $2 \mathrm{mg} / \mathrm{mL}$. Amphotericin $\mathrm{B}$ was used as a positive control at the same concentration. The fungal spore was harvested with $0.1 \%$ Tween 80 and final spore suspensions (approximately $1 \times 10^{4}$ spores $/ \mathrm{mL}$ ) were added to each well of a sterile 96 -well microtiter plate. The fungi were incubated on Potato-Dextrose broth at $28^{\circ} \mathrm{C}$ for two days and observed by the naked eye to determine the lowest concentration that inhibited fungal growth.

\section{Results}

\subsection{Microbial Isolation from the Dung Beetle and Brood Balls}

Microbial isolation from the digestive tracts of female beetles and brood balls yielded 256 bacterial isolates (119 strains from the beetle's digestive tracts and 137 strains from the brood balls) and seven fungal isolates (from the brood balls) on types of four selective agar media. Two hundred and fifty-six isolates were again simplified to 120 strains morphologically. The isolated bacterial strains showed a high diversity, but there was no significant difference between the two bacterial groups that originated from dung beetles and brood balls in morphological and molecular data.

\subsection{Antifungal Activities of Isolates Derived from the Dung Beetle}

Initial screening to evaluate the antifungal activity of the bacterial isolates sorted out 16 strains from 120 morphologically different strains (13\% of screened isolates), showing antifungal activity against at least one of the fungal strains AF1-AF4 tested (Figure 2). Moreover, these selected sixteen isolates exhibited broad-spectrum antifungal activities (Figure 2) against additional fungal strains AF5-AF11 isolated from the brood balls. They 
showed clear inhibition zones against the tested fungi (Figure 1c), whereas the inactive isolates were covered by the fungal mycelium within three to five days (Figure 1b). Five strains (AT17, AT33, AT57, AT67, and AT99) were found to possess potent and broadspectrum antifungal activities with larger inhibition zones (on average $>8 \mathrm{~mm}$ in size) against all the tested fungi (Figure 2). Above all, strain AT67 exhibited the most pronounced antifungal activity against all the tested fungi. Except for strain AT17 belong to Burkholderia sp., all the antifungal strains showed typical morphological features of streptomycetes, such as an opaque rough surface, characteristic color, and non-spreading (Figure 3a).

\begin{tabular}{|c|c|c|c|c|c|c|c|c|c|c|c|c|c|c|}
\hline \multirow{2}{*}{$\begin{array}{l}\text { Strain } \\
\text { Code }\end{array}$} & \multirow{2}{*}{$\begin{array}{l}\text { Isolated } \\
\text { Source }\end{array}$} & \multirow[t]{2}{*}{ Closest relative (NCBI accession \#) } & \multirow{2}{*}{$\begin{array}{c}\% \text { Sequence } \\
\text { Identity (query } \\
\text { coverage) }\end{array}$} & \multicolumn{11}{|c|}{$\begin{array}{l}\text { Antifungal activity (Inhibition zone size) } \\
\square<1 \mathrm{~mm}, \square=1-5 \mathrm{~mm}, \square=5-10 \mathrm{~mm}, \square>10 \mathrm{~mm}\end{array}$} \\
\hline & & & & $\mathrm{AF} 1$ & $\mathrm{AF} 2$ & AF3 & AF4 & AF5 & AF6 & AF7 & AF8 & AF9 & $\mathrm{AF} 10$ & AF11 \\
\hline AT33 & Female & S. sanglieri NBRC 100784(NR_041417) & $99.50(95)$ & & & & & & & & & & & \\
\hline AT57 & Female & S. sanglieri NBRC 100784 (NR_041417) & $99.23(99)$ & & & & & & & & & & & \\
\hline AT67 & Female & S. sanglieri NBRC 100784(NR_041417) & $99.36(97)$ & & & & & & & & & & & \\
\hline AT20 & Female & S. atratus NBRC 3897 (NR_112503) & $99.93(97)$ & & & & & & & & & & & \\
\hline AT92 & Brood ball & S. cavourensis NBRC 13026 (NR_112345) & $100(100)$ & & & & & & & & & & & \\
\hline AT60 & Brood ball & S. praecox CSSP720 (NR_115437) & $100(99)$ & & & & & & & & & & & \\
\hline AT95 & Brood ball & S. praecox CSSP720 (NR_115437) & $100(97)$ & & & & & & & & & & & \\
\hline AT120 & Female & S. praecox $\operatorname{CSSP} 720$ (NR_115437) & $99.93(99)$ & & & & & & & & & & & \\
\hline AT59 & Brood ball & S. sampsonii NBRC 13083 (NR_112362) & $99.44(99)$ & & & & & & & & & & & \\
\hline AT98 & Larva & S. sampsonii NBRC 13083 (NR_112362) & $99.57(98)$ & & & & & & & & & & & \\
\hline AT10 & Larva & S. ardesiacus NBRC 15402 (NR_112454) & $99.36(99)$ & & & & & & & & & & & \\
\hline AT13 & Brood ball & S. ardesiacus NBRC 15402 (NR_112454) & $99.15(97)$ & & & & & & & & & & & \\
\hline AT14 & Brood ball & S. ardesiacus NBRC 15402 (NR_112454) & $99.50(95)$ & & & & & & & & & & & \\
\hline AT28 & Brood ball & S. ardesiacus NBRC 15402 (NR_112454) & $99.30(99)$ & & & & & & & & & & & \\
\hline AT99 & Brood ball & S. termitum NBRC 13087 (NR_041112) & $98.88(96)$ & & & & & & & & & & & \\
\hline AT17 & Female & Burkholderiacontaminans J2956(NR_104978) & $99.86(97)$ & & & & & & & & & & & \\
\hline
\end{tabular}

Figure 2. Identification and activity spectrum of antifungal bacterial strains isolated from the dung beetle, C. tripartitus. The bacterial isolates determined by primary antifungal screening were identified by the partial 16S rRNA gene sequences amplified using the universal primers $27 \mathrm{~F}$ and 1492R. The antifungal activity against 11 fungi was evaluated by measuring inhibition zone size in the dual culture assays. The tested fungi were as follow: AF1: Aspergillus fumigatus, AF2: A. flavus, AF3: Beauveria bassiana, AF4: Metarhizium anisopliae, AF5: Trametes sp., AF6: Fusarium sp., AF7: Penicillium sp., AF8: Cladosporium sp., AF9: Penicillium sp., AF10: Aspergillus sp., AF11: Metarhizium sp. 


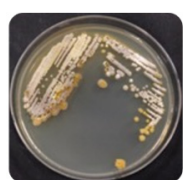

AT 10

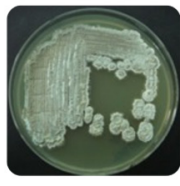

AT20

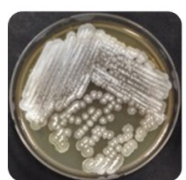

AT59

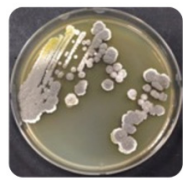

AT95

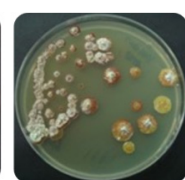

AT 13

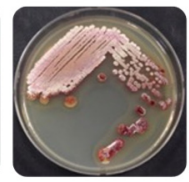

AT28

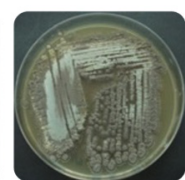

AT 60

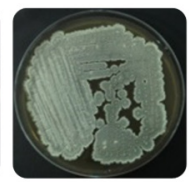

AT98

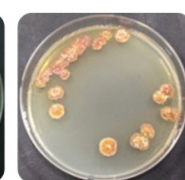

AT 14

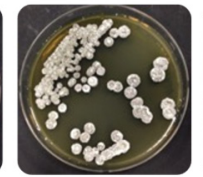

AT33

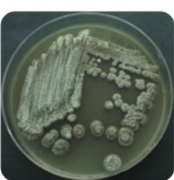

AT67

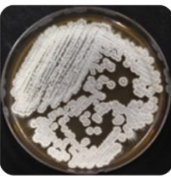

AT99

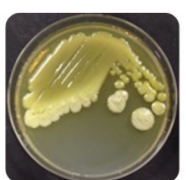

AT 17

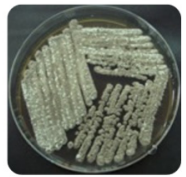

AT57

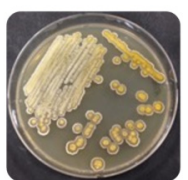

AT92

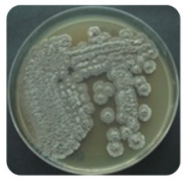

AT 120

(a)

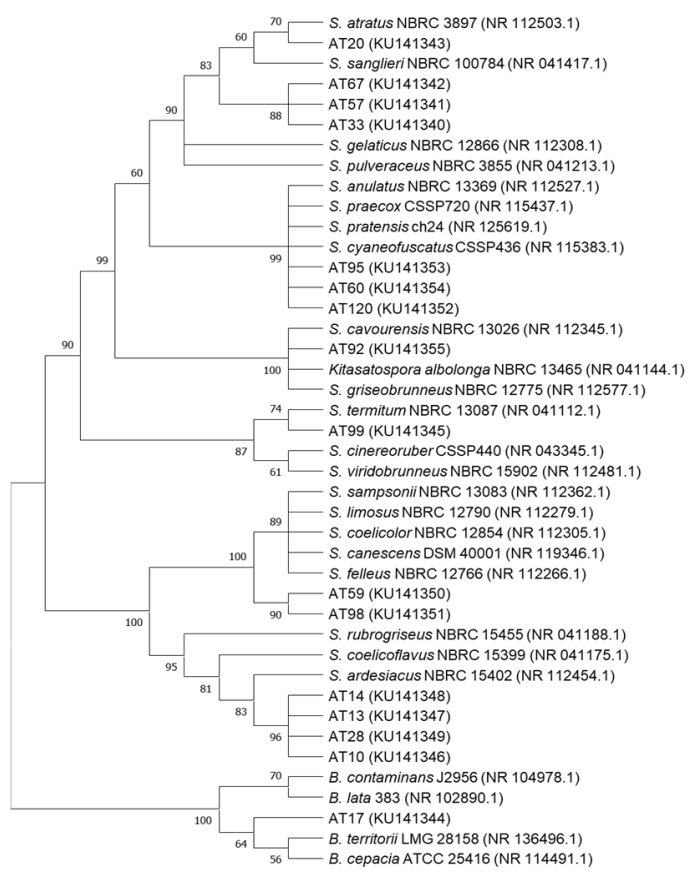

(b)

Figure 3. Antifungal bacterial strains isolated from dung beetles and brood balls. (a) Morphological characteristics of the 16 antifungal bacterial strains on YME agar plate after 20 days of cultivation. (b) Phylogenetic tree by Maximum Likelihood method based on the partial 16S rRNA gene of the 16 antifungal bacterial isolates and the close related members. Bootstrap values ( $>50 \%$ ) obtained with 1000 re-samplings are given at the branch-points and branches in less than $50 \%$ are collapsed. GenBank accession numbers are indicated in parentheses. Bar, $0.02 \mathrm{nt}$ substitutions per site.

\subsection{Identification and Phylogenetic Analysis of Antifungal Isolates}

The 16 antifungal strains were identified at the species level by $16 \mathrm{~S}$ rRNA gene sequence analysis, and the results are presented in Figure 3. According to the comparative analysis in the GenBank database, the sequence similarities to their closest phylogenetic relative showed high values ranging from 99 to $100 \%$. A phylogenetic tree was constructed using $16 \mathrm{~S}$ rRNA sequences of the 16 strains and their close neighbor strains with similarity (Table S2). The tree was created by maximum likelihood method under general time reversible model with a discrete gamma distribution and invariable sites model $(G T R+G+I)$ calculated as best model by Mega-X. The phylogenetic tree based on $16 \mathrm{~S}$ rRNA sequences revealed that all isolates, except for AT17, were clustered with their corresponding reference strain with bootstrap resampling values higher than $50 \%$ and belonged to the genus Streptomyces (Figure 3b), indicating that streptomycetes are highly associated with these dung beetles. The isolates were divided into eight groups at distinct branches as follows: Strains (1) AT33, AT57, and AT67; (2) AT20; (3) AT60, AT95, and AT120; (4) AT92; (5) AT99; (6) AT59 and AT98; (7) AT10, AT13, AT14, and AT28; and (8) AT17. These obtained 16 sequences have been submitted to the NCBI GenBank database under accession numbers KU141340 to KU141355, respectively. In order to verify that the antifungal isolates are included in a specific genus, the $r p o B$ gene sequence was prepared. From BLAST search with $540 \mathrm{bp}$ DNA corresponding to partial rpoB gene, all of the relevant strains were confirmed to belong to Streptomyces, except for AT17, which was classified as Bukholderia (Table S2). AT95 showed high similarity of $99.81 \%$ with Kitasatospora papulosa NRRL B-16504, which was recently demonstrated as a member of the species $S$. prentensis by multi-locus sequence analysis [38]. Further phylogenetic analysis of the antifungal strains and close strains based on the concatenated sequences of $16 \mathrm{~S}$ rRNA and $r p o B$ genes also showed the analogous to Figure $3 \mathrm{~b}$ clustering into eight groups except for independently located AT95 from AT60 and AT120 (Figure S2). 
Seven fungal strains isolated from the brood balls were also identified at the genus or species level by morphological imaging and ITS sequencing data. According to BLAST searches of the GenBank database, they were identified as potential entomopathogens as follows: Trametes sp. (AF5), Fusarium sp. (AF6), Penicillium sp. (AF7), Cladosporium sp. (AF8), Penicillium sp. (AF9), Aspergillus sp. (AF10) and Metarhizium sp. (AF11). The fungal strains AF10 and AF11 belonged to the same genotype group as those that were used in initial screening for antifungal activity (AF2: A. flavus ATCC 16,883 and AF4: M. anisopliae ATCC 20500).

\subsection{Association between the Dung Beetle and Antifungal Isolates}

To investigate the symbiotic association of the bacterial isolates with antifungal activity in the dung beetle ecosystem, the PCR-DGGE method was tried but there was difficulty in amplifying the $16 \mathrm{~S}$ rRNA gene of bacterial strains belonging to the genus Streptomyces from environmental samples because the group has a high genomic $G+C$ content and a low abundance, which leads to poor amplification efficiency in a competitive PCR [35]. Initial DGGE analysis on the $16 \mathrm{~S}$ V3 region amplified with universal bacterial primers (341F/GC and 518R) revealed only the presence of Enterobacteriaceae, including Citrobacter, Klebsiella, Proteus, and Serratia species in both dung beetles and brood balls (data not shown). To solve these issues, we needed to design genus-specific primer pairs for the amplification of Streptomyces $16 \mathrm{~S}$ rRNA gene. The primer DBstR was constructed based on an insertion sequence of 5 or $6 \mathrm{nt}$, which is typical of Streptomyces $16 \mathrm{~S}$ rRNA genes (Figure S3). It amplified the hypervariable V6/V7 region suitable for specific detection of Streptomyces species with a primer 984F/GC [39]. Preliminary PCR experiments using the primer pair 984F/GC and DBstR on DNA isolated from Streptomyces and non-Streptomyces strains yielded specific amplification of only Streptomyces strains, suggesting specificity in DNA amplification (Figure S3).

Though not covering all bacterial species, the DGGE profiles, which were generated with the genus-specific primer sets, consisted of abundant bands, implying that the dung beetle possessed a microbiota of multifarious bacteria related to streptomycetes. From the overall pattern of the DGGE bands, it was difficult to clearly recognize the distinguishable characteristic in the endosymbiotic bacterial community of the beetle's digestive tracts because each individual beetle showed a quite different pattern (Figures 4 and S4). In contrast, the DGGE profiles in brood balls exhibited a regular banding pattern, including several predominant bands (Figure 4). Prior to analysis, a DGGE marker was prepared including antifungal strains, AT67, AT92, AT99, AT20, and AT 95 (Figure S1). At the marker, the strains were appeared divided into two bands (band A: AT92, AT99, and AT95, band B: AT67 and AT20). In the DGGE profiles, band A and B were observed in every brood ball and some dung beetles (Figures 4 and S4).

According to these DGGE results, there is no distinct gender difference regarding the endosymbiotic bacteria corresponding to band B (Figure S4). In comparison to the DGGE profiles in the beetle's digestive tract, band B was weakly present at almost the same intensity in all brood balls (Figure 4). In addition, band B was not present in the habitat soil and cattle dung samples (Figure 2). Additionally, band A appeared in cattle dung and not in the habitat soil. Re-amplification and sequencing of each band corresponding to the band B position revealed that they had 100\% sequence similarity with the V6/V7 region in the 16S rRNA of S. sanglieri (NR_041417), which is the closest relative of strain AT67. Each band corresponding to the band A position was also identified with $100 \%$ sequence homology to S. termitum (NR_041112), which is the closest relative of strain AT99. 


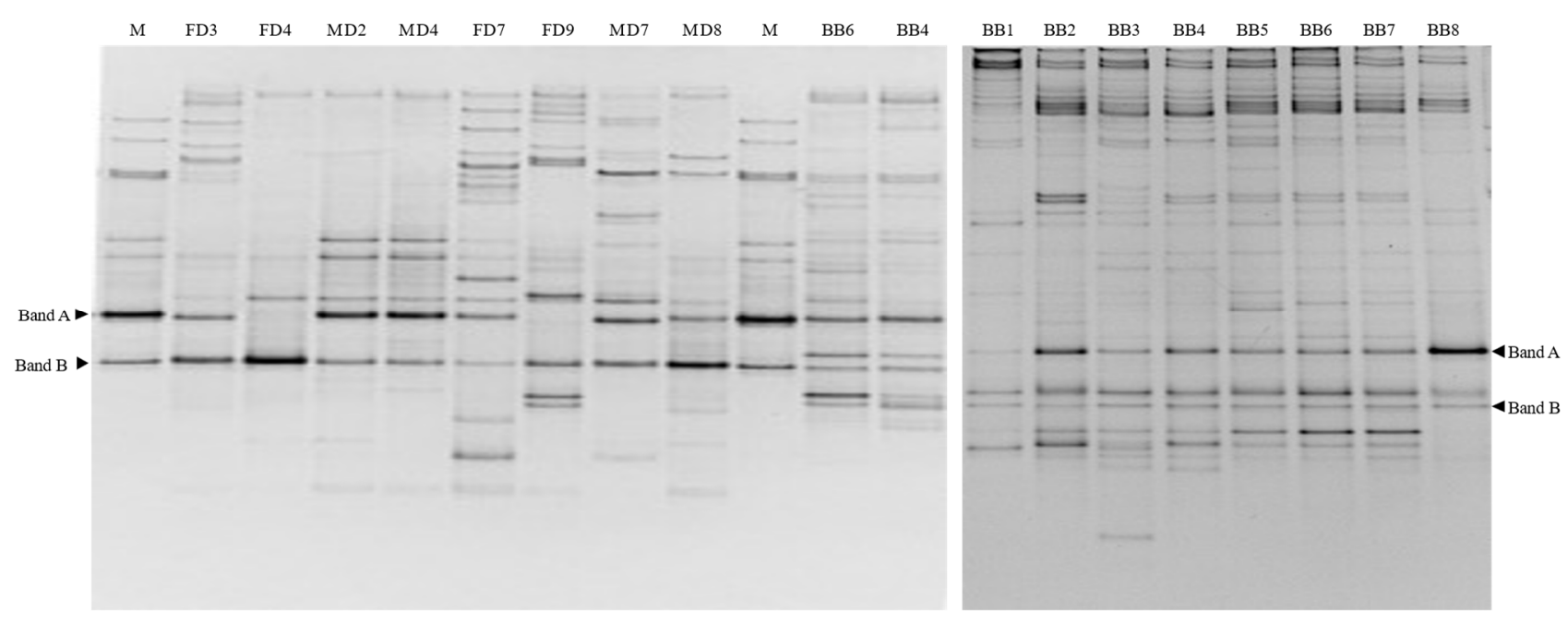

Figure 4. Representative DGGE for detection of Streptomyces antifungal isolates in samples derived from the dung beetles (left) and brood balls (right). (FD3, FD4) Digestive tracts of female dung beetles collected in 2013; (MD2, MD4) Digestive tracts of male dung beetles collected in 2013; (FD7, FD9) Digestive tracts of female dung beetles collected in 2014; (MD7, MD8) Digestive tracts of male dung beetles collected in 2014; (BB1-BB8) Brood ball; M: Marker (Band A: AT92, AT95 and AT99, Band B: AT20 and AT67]).

\subsection{Chemical Profile of 16 Antifungal Strains}

HPLC chromatograms of culture extracts from the 16 antifungal strains are summarized in Figure 5. The comparative metabolic analysis revealed that the strains that clustered in the same phylogenetic branch showed a similar metabolic profile with a slight difference in the relative peak intensities (Figure 5). For instance, strains AT33, AT57, and AT67 clustered together in a branch presented an almost similar metabolic pattern, but the intensity of each peak was slightly different, while Burkholderia sp. AT17 in the discrete branch exhibited a completely different metabolic pattern. Similarity analysis of the HPLC chromatograms with BioNumerics software revealed that strains belonging to the almost identical species level were clustered together with a high similarity percentage and exhibited similar clustering to those of the phylogenetic tree analysis based on their $16 \mathrm{~S}$ rRNA sequences (Figures $3 \mathrm{~b}$ and 5 ). This implies that the secondary metabolites profile might be also useful for bacterial chemotaxonomic classification. 


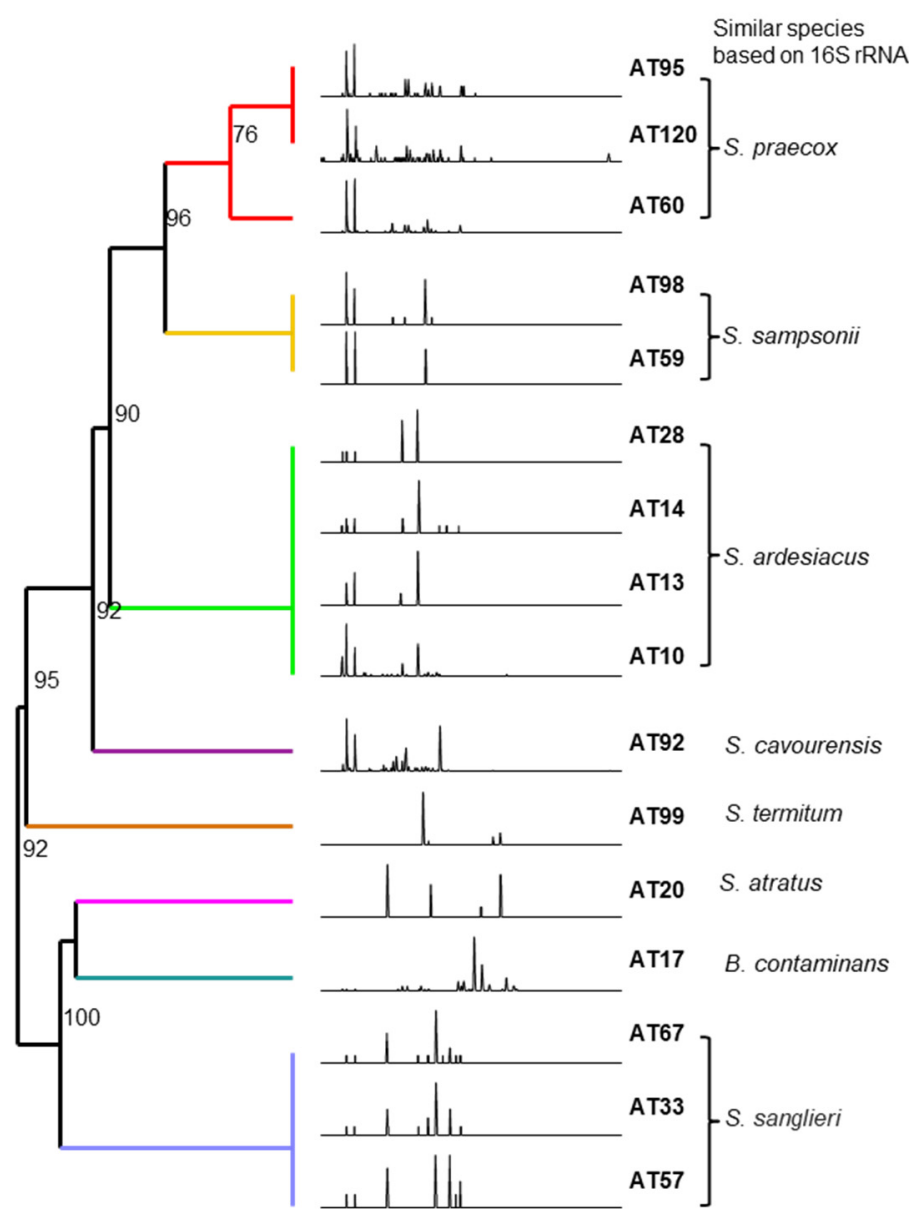

Figure 5. Similarity dendrogram constructed based on HPLC chromatograms with the Dice coefficient and UPGMA method using BioNumerics software.

\subsection{Antifungal Metabolite Produced by Strain AT67}

Further LC-MS analysis was executed to identify the actual antifungal metabolites produced from the most potent antifungal strain AT67 and revealed the presence of a major component in the culture extract (Figure 6). The component was thought to be a polyene macrocyclic lactam through pre-screening using our in-house HPLC-MS-UV database and identified as sceliphrolactam by the comparison with previously reported HPLC and MS data (Figure 6) [40,41]. In the MIC assay, the crude extract and the three fractions (Fr3, Fr4, and Fr5) derived from AT67 showed inhibitory activity against the fungal strains AF10 and AF11 with MIC values of $0.125-0.5 \mathrm{mg} / \mathrm{mL}$. The active fractions showed a slight difference in activity level according to the content of sceliphrolactam in the fractions. Additionally, the compound was detected in all of the inhibitory zones formed between strain AT67 and the fungi tested in dual culture assay (Figure 6). Therefore, sceliphrolactam was presumed to be a putative candidate produced from strain AT67 as an active compound against pathogenic fungi. 


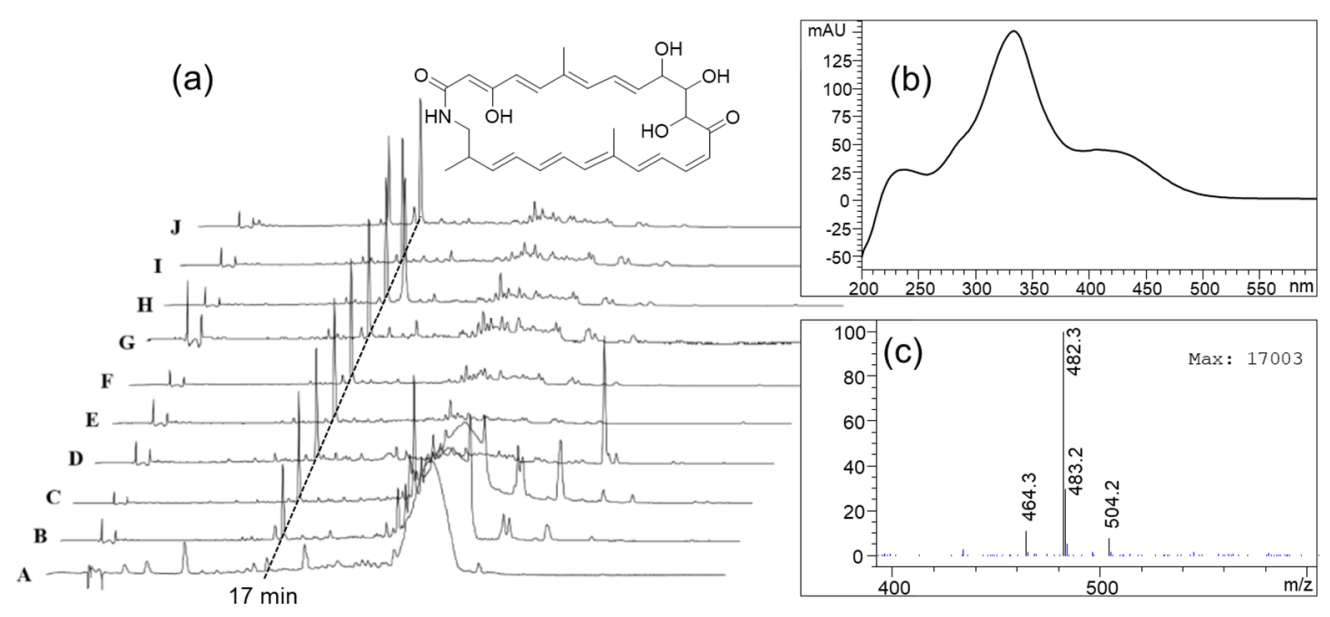

Figure 6. Antifungal compound, sceliphrolactam produced by AT67. (a) HPLC chromatograms derived from extracts of culture and inhibition zone (IZ) on agar plates of dual culture between AT67 and fungal strains (A: broth culture of AT67, B: Agar culture of AT67, C: IZ between AT67 and AF1, D: IZ between AT67 and AF2, E: IZ between AT67 and AF5, F: IZ between AT67 and AF6, G: IZ between AT67 and AF7, H: IZ between AT67 and AF9, I: IZ between AT67 and AF10, J: IZ between AT67 and AF11.). UV absorption (b) and mass spectrum (c) of the peak at a retention time of 17 min (UV maxima at 333 and $420 \mathrm{~nm}, \mathrm{~m} / \mathrm{z} 482.3$ for $[\mathrm{M}+\mathrm{H}]^{+}$and $m / z 504.2$ for $\left.[\mathrm{M}+\mathrm{Na}]^{+}\right)$, indicating sceliprolactam.

\section{Discussion}

Dung beetles spend most of their life close to a fecal-rich environment. Due to this unique lifestyle, they are threatened by antagonists such as entomopathogenic fungi isolated abundantly from dung balls. These fungi invade the nutrient-rich hemolymph of the insect host by using a combination of cuticle-degrading enzymes and mechanical force, eventually causing the death of the host [42,43]. Moreover, these entomopathogens can easily penetrate the insect eggs and larvae $[44,45]$. Thus, the beetles need a counterplan to care for their offspring. According to previous reports, e.g., when the dung beetle $C$. tripartitus is invaded by microbial pathogens, it defends itself by using an antimicrobial peptide Coprisin as a defensive immune response [46,47]. In this study, we postulated an antifungal bacterial symbiont as a defensive agent against fungal invasion in the brood care of the dung beetle $C$. tripartitus and tried to demonstrate this through culture-dependent and independent approaches.

Brood care by the dung beetle causes frequent and consistent interaction via oral secretion from the digestive tract, between the beetle and brood ball; we, thus, focused on the antifungal bacterial association derived from microbiota in dung beetles and brood balls. In this study, we revealed that most of the bacterial strains showing remarkable antifungal activity belong to Streptomyces spp., which are often referred to as protective symbionts in the insect-bacteria symbiosis $[16,40]$. The consistent presence of antifungal streptomycetes in the dung beetles $C$. tripartitus was evaluated by culture independent method using PCR-DGGE with primer set designed for Streptomyces 16S rRNA genes. This allowed us to assess the relative proportions and distribution of antifungal Streptomyces isolates through a semi-quantitative analysis based on the DGGE bands and showed that Streptomyces sp. AT67 and AT99 were common and major species in the brood balls and digestive tract of $C$. tripartitus. However, DGGE results for the habitat soil or cattle dung suggested a different origination of the two species. The AT67 strain did not occur in the habitat soil and cattle dung, which are the main materials for the construction of brood balls, whereas the AT99 strain was present as a major species in the cattle dung, indicating that the AT99 strain is an external organism present in the dung beetle's digestive system introduced by feeding. On the other hand, the presence of strain AT67 in only the brood balls and digestive tract of the dung beetles, but not in the soil or cattle dung, suggests that it may be an endosymbiont of the dung beetles. In addition, the successive 
occurrences of strain AT67 over three years suggest that C. tripartitus vertically transmits the endosymbiotic bacterium to their offspring via brood balls, which are the sole food source for the developing offspring. Recently, vertical transmission of microbiota via the food carcass has been shown in burying beetles [48-50]. Similarly, C. tripartitus may utilize the endosymbionts stored in its digestive system during the construction and maternal care of their brood balls.

Along with the plausible symbiotic association, mainly with streptomycetes, we ascertained that the potent antifungal strain AT67 produced a diffusible antifungal compound, sceliphrolactam, as a major secondary metabolite. The compound was first isolated from wasp-associated Streptomyces sp. and reported to possess antifungal activity against amphotericin B-resistant Candida albicans (MIC $=4 \mu \mathrm{g} / \mathrm{mL}, 8.3 \mu \mathrm{M}$ ). Sceliphrolactam is a polyene macrocyclic lactam and its structure is extremely fragile and labile [41]. Therefore, exposures of the compound to the environments with changeable conditions often lead to its degradation, which may consequently lead to loss of its antifungal activity [51]. Considering orphaned broods without continuous brood ball care are unsuccessful in surviving due to the parasite and pathogen invasion, there may be a link between continuous brood ball care and the antifungal compound production by streptomyces sp.

On the other hands, fungi are mainly described as entomopathogen, but they often involves a mutualistic players in insect-microbe symbiosis such as Fusarium solani, which is cultivated by a species of ambrosia beetle Xylosandrus compactus as nutrition source [52], and Leucoagaricus gongylophorus, which provides food to the ant Acromyrmex octospinosus [53]. In addition, one clade of ambrosia beetles has evolved with a mutualist of the polypore fungus Flavodon ambrosius facilitating wood decay by lignin-modifying enzymes [54]. Therefore, we need to investigate more systemically the fungi isolated from the brood balls to reveal their roles and functions in the insect ecosystem by further study.

To sum up these results, the dung beetle C. tripartitus harbors antifungal bacteria, which are mainly comprised of Streptomyces bacteria that are highly likely to protect its offspring in brood balls from diverse fungal pathogens. Furthermore, as antifungal symbionts, the Streptomyces sp. AT67 was proposed to play a significant role as a defensive agent in dung beetle-bacteria symbiosis. These defensive associations may be maintained through successful transmission to the brood ball and production of antifungal substances as a major secondary metabolite.

Supplementary Materials: The following are available online at https:/ / www.mdpi.com/article / 10.3390 / microorganisms9091980/s1, Figure S1: DGGE marker (M) used in this study for the rapid identification of Streptomyces antifungal isolates (upper) and DGGE for the detection of Streptomyces antifungal isolates from the habitat soil and cattle dung., Figure S2: Phylogenetic tree based on the concatenated sequences of $16 \mathrm{~S}$ rRNA and rpoB genes., Figure S3: PCR result using genus-specific primer sets, Figure S4: DGGE for detection of Streptomyces antifungal isolates from the digestive tracts of the dung beetles collected in 2013 and 2014., Table S1: Primer sets used for PCR-DGGE, Table S2: BLASTN search results with 16S rRNA and rpoB sequences.

Author Contributions: Conceptualization, H.C.K.; Data curation, S.H.K.; formal analysis and validation, S.H.K. and G.P.; investigation, writing—original draft preparation, S.H.K. and J.-S.P.; writingreview and editing, G.P., J.-S.P. and H.C.K.; funding acquisition, H.C.K.; Project administration and Resources, H.C.K. All authors have read and agreed to the published version of the manuscript.

Funding: This work was supported by intramural grants (2E31300 and 2Z06482) from the Korea Institute of Science and Technology, Republic of Korea.

Institutional Review Board Statement: Not applicable.

Informed Consent Statement: Not applicable.

Data Availability Statement: Not applicable.

Acknowledgments: We give special thanks to Hea Son Bang (National Institute of Agricultural Science, Department of Rural Administration, Republic of Korea) for her help to provide the brood balls. 
Conflicts of Interest: The authors declare no conflict of interest. The funders had no role in the design of the study; in the collection, analyses, or interpretation of data; in the writing of the manuscript, or in the decision to publish the results.

\section{References}

1. Bang, H.-S.; Crespo, C.H.; Na, Y.-E.; Han, M.-S.; Lee, J.-H. Reproductive development and seasonal activity of two Korean native Coprini species (Coleoptera: Scarabaeidae). J. Asia-Pac. Èntomol. 2008, 11, 195-199. [CrossRef]

2. Bang, H.S.; Lee, J.-H.; Kwon, O.S.; Na, Y.E.; Jang, Y.S.; Kim, W.H. Effects of paracoprid dung beetles (Coleoptera: Scarabaeidae) on the growth of pasture herbage and on the underlying soil. Appl. Soil Ecol. 2005, 29, 165-171. [CrossRef]

3. Hanski, I.; Cambefort, Y. Dung Beetle Ecology; Princeton University Press: Princeton, NJ, USA, 1991; pp. $481-486$.

4. Scholtz, C.H.; Davis, A.L.V.; Kryger, U. Evolutionary Biology and Conservation of Dung Beetles; Pensoft Sofia-Moscow: Moscow, Russia, 2009.

5. Bang, H.S.; Huerta, C.; Kim, J.I.; Goo, T.W. Studies on the Ecology of Oviposition of Copris tripartitus Waterhouse and Copris ochus (Motschulsky) (Coleoptera: Scarabaeidae). Korean J. Entomol. 2001, 31, 237-242.

6. Tyndale-Biscoe, M. Adaptive significance of brood care of Copris diversus Waterhouse (Coleoptera: Scarabaeidae). Bull. Èntomol. Res. 1984, 74, 453-461. [CrossRef]

7. Halffter, G.; Huerta, C.; Lopez-Portillo, J. Parental care and offspring survival inCopris incertusSay, a sub-social beetle. Anim. Behav. 1996, 52, 133-139. [CrossRef]

8. Halffter, G.; Edmonds, W.D. The nesting behavior of dung beetles (Scarabaeinae). In An Ecological and Evolutive Approach. The Nesting Behavior of Dung Beetles (Scarabaeinae); Instituto de Ecologia: Ciudad de Mexico, Mexico, 1982.

9. Byrne, M.J.; Watkins, B.; Bouwer, G. Do dung beetle larvae need microbial symbionts from their parents to feed on dung? Ecol. Entomol. 2013, 38, 250-257. [CrossRef]

10. Florez, L.V.; Biedermann, P.; Engl, T.; Kaltenpoth, M. Defensive symbioses of animals with prokaryotic and eukaryotic microorganisms. Nat. Prod. Rep. 2015, 32, 904-936. [CrossRef]

11. Currie, C.R.; Scott, J.; Summerbell, R.C.; Malloch, D. Fungus-growing ants use antibiotic-producing bacteria to control garden parasites. Nature 1999, 398, 701-704. [CrossRef]

12. Kaltenpoth, M.; Goettler, W.; Dale, C.; Stubblefield, J.W.; Herzner, G.; Roeser-Mueller, K.; Strohm, E. 'Candidatus Streptomyces philanthi', an endosymbiotic streptomycete in the antennae of Philanthus digger wasps. Int. J. Syst. Evol. Microbiol. 2006, 56, 1403-1411. [CrossRef] [PubMed]

13. Scott, J.; Oh, D.-C.; Yuceer, M.C.; Klepzig, K.D.; Clardy, J.; Currie, C.R. Bacterial Protection of Beetle-Fungus Mutualism. Science 2008, 322, 63. [CrossRef] [PubMed]

14. Vasquez, A.; Forsgren, E.; Fries, I.; Paxton, R.J.; Flaberg, E.; Szekely, L.; Olofsson, T.C. Symbionts as Major Modulators of Insect Health: Lactic Acid Bacteria and Honeybees. PLoS ONE 2012, 7, e33188. [CrossRef]

15. Oh, D.-C.; Poulsen, M.; Currie, C.R.; Clardy, J. Dentigerumycin: A bacterial mediator of an ant-fungus symbiosis. Nat. Chem. Biol. 2009, 5, 391-393. [CrossRef] [PubMed]

16. Kroiss, J.; Kaltenpoth, M.; Schneider, B.; Schwinger, M.-G.; Hertweck, C.; Maddula, R.K.; Strohm, E.; Svatos, A. Symbiotic streptomycetes provide antibiotic combination prophylaxis for wasp offspring. Nat. Chem. Biol. 2010, 6, 261-263. [CrossRef]

17. Van Arnam, E.B.; Currie, C.R.; Clardy, J. Defense contracts: Molecular protection in insect-microbe symbioses. Chem. Soc. Rev. 2017, 47, 1638-1651. [CrossRef]

18. Estes, A.M.; Hearn, D.J.; Snell-Rood, E.C.; Feindler, M.; Feeser, K.; Abebe, T.; Hotopp, J.C.D.; Moczek, A.P. Brood Ball-Mediated Transmission of Microbiome Members in the Dung Beetle, Onthophagus taurus (Coleoptera: Scarabaeidae). PLoS ONE 2013, 8 , e79061. [CrossRef]

19. Kim, S.-H.; Ko, H.; Bang, H.-S.; Park, S.-H.; Kim, D.-G.; Kwon, H.C.; Kim, S.Y.; Shin, J.; Oh, D.-C. Coprismycins A and B, neuroprotective phenylpyridines from the dung beetle-associated bacterium, Streptomyces sp. Bioorganic Med. Chem. Lett. 2011, 21, 5715-5718. [CrossRef]

20. Park, S.-H.; Moon, K.; Bang, H.-S.; Kim, S.-H.; Kim, D.-G.; Oh, K.-B.; Shin, J.; Oh, D.-C. Tripartilactam, a Cyclobutane-Bearing Tricyclic Lactam from a Streptomyces sp. in a Dung Beetle's Brood Ball. Org. Lett. 2012, 14, 1258-1261. [CrossRef] [PubMed]

21. Kim, S.-H.; Kwon, S.H.; Park, S.-H.; Lee, J.K.; Bang, H.-S.; Nam, S.-J.; Kwon, H.C.; Shin, J.; Oh, D.-C. Tripartin, a Histone Demethylase Inhibitor from a Bacterium Associated with a Dung Beetle Larva. Org. Lett. 2013, 15, 1834-1837. [CrossRef]

22. Um, S.; Bang, H.-S.; Shin, J.; Oh, D.-C. Actinofuranone C, a new 3-furanone-bearing polyketide from a dung beetle-associated bacterium. Nat. Prod. Sci. 2013, 19, 71-75.

23. Um, S.; Shin, J.; Oh, K. New bioactive secondary metabolites from dung beetle-associated bacteria. Planta Medica 2016, 81, S1-S381. [CrossRef]

24. Tyndale-Biscoe, M.; Wallace, M.M.H.; Walker, J.M. An ecological study of an Australian dung beetle, Onthophagus granulatus Boheman (Coleoptera: Scarabaeidae), using physiological age-grading techniques. Bull. Èntomol. Res. 1981, 71, 137-152. [CrossRef]

25. Huerta, C.; Bang, H.S. Fecundity and Offspring Survival of Copris tripartitus Waterhouse (Coleoptera, Scarabaeidae: Scarabaeinae) Under Laboratory Rearing Conditions. Coleopt. Bull. 2004, 58, 501-507. [CrossRef]

26. Lane, D.J. 16S/23S rRNA sequencing. In Nucleic Acid Techniques in Bacterial Systems; Jhon Wiley \& Sons: Hoboken, NJ, USA, 1991. 
27. White, T.; Bruns, T.; Lee, S.; Taylor, J. Amplification and Direct Sequencing Of Fungal Ribosomal Rna Genes For Phylogenetics; Jhon Wiley \& Sons: Hoboken, NJ, USA, 1990; pp. 315-322. [CrossRef]

28. Hall, T.A. BioEdit: A user-friendly biological sequence alignment editor and analysis program for Windows 95/98/NT. Nucleic Acid Symp. Ser. 1999, 41, 95-98.

29. Zhang, Z.; Schwartz, S.; Wagner, L.; Miller, W. A Greedy Algorithm for Aligning DNA Sequences. J. Comput. Biol. 2000, 7, 203-214. [CrossRef]

30. Thompson, J.D.; Higgins, D.; Gibson, T.J. CLUSTAL W: Improving the sensitivity of progressive multiple sequence alignment through sequence weighting, position-specific gap penalties and weight matrix choice. Nucleic Acids Res. 1994, 22, 4673-4680. [CrossRef] [PubMed]

31. Kumar, S.; Tamura, K.; Nei, M. MEGA: Molecular Evolutionary Genetics Analysis software for microcomputers. Bioinformatics 1994, 10, 189-191. [CrossRef] [PubMed]

32. Nei, M.; Kumar, S. Molecular Evolution and Phylogenetics; Oxford University Press: Oxford, UK, 2000.

33. Thong, W.L.; Shin-Ya, K.; Nishiyama, M.; Kuzuyama, T. Methylbenzene-Containing Polyketides from a Streptomyces that Spontaneously Acquired Rifampicin Resistance: Structural Elucidation and Biosynthesis. J. Nat. Prod. 2016, 79, 857-864. [CrossRef]

34. Barke, J.; Seipke, R.F.; Grüschow, S.; Heavens, D.; Drou, N.; Bibb, M.J.; Goss, R.J.; Yu, D.W.; I Hutchings, M. A mixed community of actinomycetes produce multiple antibiotics for the fungus farming ant Acromyrmex octospinosus. BMC Biol. $2010,8,109$. [CrossRef]

35. Heuer, H.; Krsek, M.; Baker, P.; Smalla, K.; Wellington, E.M. Analysis of actinomycete communities by specific amplification of genes encoding $16 \mathrm{~S}$ rRNA and gel-electrophoretic separation in denaturing gradients. Appl. Environ. Microbiol. 1997, 63, 3233-3241. [CrossRef]

36. Sheffield, V.C.; Cox, D.R.; Lerman, L.S.; Myers, R.M. Attachment of a 40-base-pair G + C-rich sequence (GC-clamp) to genomic DNA fragments by the polymerase chain reaction results in improved detection of single-base changes. Proc. Natl. Acad. Sci. USA 1989, 86, 232-236. [CrossRef]

37. Sneath, P.H.A.; Sokal, R.R. Numerical Taxonomy. The Principles and Practice of Numerical Classification; W. H. Freeman and Co.: San Francisco, CA, USA, 1973.

38. Labeda, D.P.; Dunlap, C.; Rong, X.; Huang, Y.; Doroghazi, J.R.; Ju, K.-S.; Metcalf, W.W. Phylogenetic relationships in the family Streptomycetaceae using multi-locus sequence analysis. Antonie van Leeuwenhoek 2016, 110, 563-583. [CrossRef] [PubMed]

39. Mehling, A.; Wehmeier, U.F.; Piepersberg, W. Nucleotide sequences of streptomycete 16S ribosomal DNA: Towards a specific identification system for streptomycetes using PCR. Microbiology 1995, 141, 2139-2147. [CrossRef] [PubMed]

40. Poulsen, M.; Oh, D.-C.; Clardy, J.; Currie, C.R. Chemical Analyses of Wasp-Associated Streptomyces Bacteria Reveal a Prolific Potential for Natural Products Discovery. PLoS ONE 2011, 6, e16763. [CrossRef]

41. Oh, D.-C.; Poulsen, M.; Currie, C.R.; Clardy, J. Sceliphrolactam, a Polyene Macrocyclic Lactam from a Wasp-Associated Streptomyces sp. Org. Lett. 2011, 13, 752-755. [CrossRef] [PubMed]

42. Leger, R.S.; Cooper, R.; Charnley, A. Cuticle-degrading enzymes of entomopathogenic fungi: Cuticle degradation in vitro by enzymes from entomopathogens. J. Invertebr. Pathol. 1986, 47, 167-177. [CrossRef]

43. Butt, T.M.; Ibrahim, L.; Clark, S.J.; Beckett, A. The Germination Behavior of Metarhizium-Anisopliae on the Surface of Aphid and Flea Beetle Cuticles. Mycol. Res. 1995, 99, 945-950. [CrossRef]

44. Steinkraus, D.C.; Geden, C.J.; Rutz, D.A. Susceptibility of Lesser Mealworm (Coleoptera, Tenebrionidae) to Beauveria-Bassiana (Moniliales, Moniliaceae)—Effects of Host Stage, Substrate, Formulation, and Host Passage. J. Med. Entomol. 1991, 28, 314-321. [CrossRef]

45. Gindin, G.; Levski, S.; Glazer, I.; Soroker, V. Evaluation of the entomopathogenic fungiMetarhizium anisopliae andBeauveria bassiana against the red palm weevilRhynchophorus ferrugineus. Phytoparasitica 2006, 34, 370-379. [CrossRef]

46. Hwang, J.-S.; Lee, J.; Kim, Y.-J.; Bang, H.-S.; Yun, E.-Y.; Kim, S.-R.; Suh, H.-J.; Kang, B.R.; Nam, S.-H.; Jeon, J.-P.; et al. Isolation and Characterization of a Defensin-Like Peptide (Coprisin) from the Dung Beetle, Copris tripartitus. Int. J. Pept. 2009, $2009,1-5$. [CrossRef]

47. Lee, J.; Hwang, J.-S.; Hwang, I.-S.; Cho, J.; Lee, E.; Kim, Y.; Lee, D.G. Coprisin-induced antifungal effects in Candida albicans correlate with apoptotic mechanisms. Free Radic. Biol. Med. 2012, 52, 2302-2311. [CrossRef]

48. Wang, Y.; Rozen, D.E. Gut Microbiota Colonization and Transmission in the Burying Beetle Nicrophorus vespilloides throughout Development. Appl. Environ. Microbiol. 2017, 83, e03250-16. [CrossRef] [PubMed]

49. Duarte, A.; Welch, M.; Swannack, C.; Wagner, J.; Kilner, R.M. Strategies for managing rival bacterial communities: Lessons from burying beetles. J. Anim. Ecol. 2017, 87, 414-427. [CrossRef]

50. Shukla, S.P.; Sanders, J.G.; Byrne, M.; Pierce, N.E. Gut microbiota of dung beetles correspond to dietary specializations of adults and larvae. Mol. Ecol. 2016, 25, 6092-6106. [CrossRef] [PubMed]

51. Hwang, S.; Kim, E.; Lee, J.; Shin, J.; Yoon, Y.J.; Oh, D.-C. Structure Revision and the Biosynthetic Pathway of Tripartilactam. J. Nat. Prod. 2020, 83, 578-583. [CrossRef]

52. Egonyu, J.P.; Torto, B. Responses of the ambrosia beetle Xylosandrus compactus (Coleoptera: Curculionidea: Scolytinae) to volatile constituents of its symbiotic fungus Fusarium solani (Hypocreales: Nectriaceae). Arthropod-Plant Interact. 2017, 12, 9-20. [CrossRef] 
53. Currie, C.R. A Community of Ants, Fungi, and Bacteria: A Multilateral Approach to Studying Symbiosis. Annu. Rev. Microbiol. 2001, 55, 357-380. [CrossRef] [PubMed]

54. Kasson, M.T.; Wickert, K.L.; Stauder, C.M.; Macias, A.M.; Berger, M.C.; Simmons, D.R.; Short, D.P.; DeVallance, D.B.; Hulcr, J. Mutualism with aggressive wood-degrading Flavodon ambrosius (Polyporales) facilitates niche expansion and communal social structure in Ambrosiophilus ambrosia beetles. Fungal Ecol. 2016, 23, 86-96. [CrossRef] 